\title{
Geodesic modes driven by untrapped resonances of NB energetic ions in tokamaks
}

Cite as: Phys. Plasmas 26, 102508 (2019); https://doi.org/10.1063/1.5110175

Submitted: 14 May 2019 . Accepted: 22 September 2019 . Published Online: 17 October 2019

A. G. Elfimov (D) R. M. O. Galvão, and N. N. Gorelenkov (D)
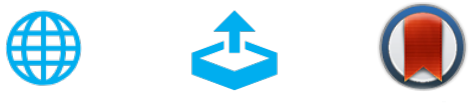

View Online

Export Citation

\section{ARTICLES YOU MAY BE INTERESTED IN}

The interplay of an external torque and ExB structure formation in tokamak plasmas Physics of Plasmas 26, 102306 (2019); https://doi.org/10.1063/1.5120903

Verification of an energetic-electron-driven $\beta$-induced Alfvén eigenmode in the HL-2A tokamak

Physics of Plasmas 26, 102507 (2019); https://doi.org/10.1063/1.5115811

Turbulent transport in TCV plasmas with positive and negative triangularity

Physics of Plasmas 26, 102302 (2019); https://doi.org/10.1063/1.5115390

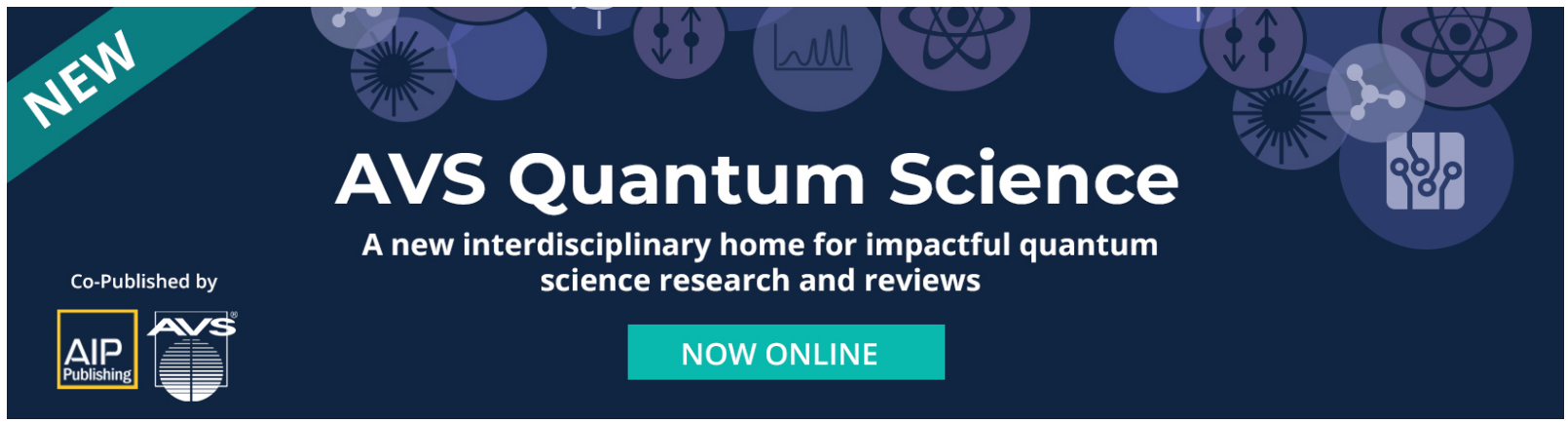




\title{
Geodesic modes driven by untrapped resonances of NB energetic ions in tokamaks
}

\author{
Cite as: Phys. Plasmas 26, 102508 (2019); doi: 10.1063/1.5110175 \\ Submitted: 14 May 2019 - Accepted: 22 September 2019 . \\ Published Online: 17 October 2019
}

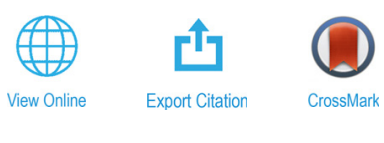

\author{
A. G. Elfimov, ${ }^{1}$ (D) R. M. O. Galvão, ${ }^{2}$ and N. N. Gorelenkov ${ }^{3}$ (iD
}

\author{
AFFILIATIONS \\ 'Institute of Physics, University of São Paulo, São Paulo 05508-090, Brazil \\ ${ }^{2}$ National Institute for Space Research, São José dos Campos 12227-010, Brazil \\ ${ }^{3}$ Princeton Plasma Physics Laboratory, Princeton University, Princeton, New Jersey 08543, USA
}

\begin{abstract}
Geodesic modes are typically excited by a minor concentration of energetic ions, but unstable mode frequencies are substantially different from Geodesic Acoustic Modes (GAMs) and are named EGAM (Energetic particle GAM). The EGAM instability driven by Neutral Beam Injection (NBI) has been observed in DIII-D tokamak experiments. The problem of the geodesic mode instability is analytically studied using a full drift kinetic equation. To analyze the instability condition, an ionization NBI location is assumed to be on the high field side of tokamaks. A minority NBI ion distribution is modeled by an energetic ion tail in the untrapped-passing region that remains between a magnetic axis and the trapped NBI boundary. The EGAM instability condition is defined by the parallel NBI ion velocity $v_{\|} \approx(1.2-1.5) \omega R_{0} q_{0}$ that has to be above the effective EGAM phase velocity. In this case, the EGAM frequency is $\approx 50 \%$ below the standard stable GAM frequency, which is reduced by a small concentration of energetic NBI ions. Qualitative comparison of the developed geodesic mode theory with NBI heating experiments in the midregion of the tokamak plasma is discussed.
\end{abstract}

Published under license by AIP Publishing. https://doi.org/10.1063/1.5110175

\section{INTRODUCTION}

Geodesic Acoustic Modes (GAMs) are $\mathrm{M}=0, \mathrm{~N}=0$ axisymmetric modes combined with $\mathrm{M}= \pm 1, \pm 2$ poloidal sidebands with frequency $^{1} \omega_{G}^{2} \approx\left(7 T_{i} / 2+2 T_{e}\right) / R_{0}^{2} m_{i}$ in hot plasmas, where $T_{e, i}$ are the electron or ion temperatures in energy units, $R_{0}$ is the major radius of the plasma column, and $m_{i}$ is the ion mass. Geodesic eigenmodes have been experimentally observed in tokamak Ohmic discharges ${ }^{2,3}$ and also detected during ion cyclotron resonance heating (ICRH) ${ }^{4}$ and Neutral Beam Injection (NBI) ${ }^{5-9}$ heating discharges in tokamaks. These modes attract attention due to accompanied energetic ion loss observed in experiments ${ }^{3-7}$ and modification of the plasma transport, as discussed in theoretical models. ${ }^{8,9}$ In the $\mathrm{ICRH}^{4}$ and NBI heating ${ }^{6,7}$ experiments, geodesic mode instabilities are usually driven by trapped ${ }^{4}$ and untrapped fast ions, respectively. ${ }^{6,7}$ According to models ${ }^{10,11}$ of the instability in discharges with NBI, the energetic particle GAM (EGAM) instability is defined by the pitch angle dependence in velocity space with a slowing-down energy distribution that is finished at the critical energy. ${ }^{12}$ This distribution is formed during the slowing time delay ${ }^{12,13} t_{\mathrm{sl}} \approx 6.3 \times 10^{8} A T_{e}^{3 / 2} / Z_{e f} n_{0} \ln \Lambda$ (where $A$ is the normalized mass of the beam particles, $Z_{e f}$ is the effective charge number, and $n_{0}$ is the plasma density) after the beginning of the NBI. It should be noted that the EGAM instability has been observed in a series of
NBI experiments ${ }^{6,7}$ after a time delay of 2-4 ms, which is much shorter than the $t_{\mathrm{sl}}$-time used in Refs. 10 and 11. The observed EGAM frequency $^{6,7}$ was found to be much smaller than the results of theoretical calculations $s^{14,15}$ of the $\omega_{E G}$-geodesic frequency driven by the beam, plasma parameters, and the angle between the magnetic field and NBI velocity. In the experiments, ${ }^{6}$ the EGAM instabilities, driven by the counter NBI, were detected at a half minor radius and deeper, which is covered by the untrapped NBI region, but these types of instabilities are not observed ${ }^{6}$ in the trapped ion region of higher radius positions ${ }^{16}$ for the injected beam. Here, it is proposed to study the EGAM instability driven by untrapped energetic ions at the beginning of NBI. This provides motivation to find a cause of the instability driven by untrapped energetic ions following a time period shorter than the $t_{\mathrm{sl}}$ time.

\section{THEORETICAL MODEL}

A full kinetic description of the periodic transit effects of untrapped ions ${ }^{16,18}$ is applied to find the GAM instability condition and its frequency modification during the NBI heating before the strong redistribution of slowing-down NBI ions. The geodesic properties of plasmas are investigated in a tokamak with the circular flux surfaces $(z=r \cdot \sin \theta)$, $R=R_{0}+r \cdot \cos \theta$, in the large aspect ratio approximation $\varepsilon=r / R_{0} \ll 1$, which are formed by a magnetic field with toroidal and poloidal 
components, $B_{\zeta}=B_{0} R_{0} / R, B_{\vartheta}=r B_{\zeta} / q R_{0} \cong 0.2 I / r$, where $B_{0}$ is the magnetic field at the magnetic axis, $q$ is the safety factor, and $I(\mathrm{~A})$ is the current propagating on equilibrium surfaces of radius $r(\mathrm{~cm})$. Here, the $\delta$ Dirac function pitch angle dependence is used in the NBI distribution

$$
\begin{aligned}
F_{b}= & C \delta\left(\lambda-\lambda_{0}\right) \mathrm{H}(1+1 / d-u) \mathrm{H}\left(\mathrm{u}-v_{c r} / v_{0}\right) \\
& \times \operatorname{erfc}\left(d\left(v_{s l} / v_{0}-u\right)\right) / 2 u^{3}
\end{aligned}
$$

that has a piecewise slowing-down energy distribution, which is limited by the $\mathrm{H}(x)$-Heaviside function and probability integral ${ }^{17}$ $\operatorname{erfc}(x)=1-\operatorname{erf}(x)$. Here, the parameter $1 / d$ is assumed to be small $1 / d^{2} \leq \varepsilon / \pi \ll 1$ defined by the small diffusion effects produced by collisions and a reducing parallel energy by electrons with the slowing-down velocity ${ }^{13} v_{s l}=v_{0}\left(1-t / t_{s l}\right)$ that is estimated during the short time delay $t \ll t_{\mathrm{sl}}$, where $v_{0}$ is the NBI velocity, and $C=n_{0} n_{b} \sqrt{1+\varepsilon-\lambda_{0}} / \pi v_{0}^{3} \ln \left(v_{0} / v_{s l}\right)$. The NBI transit frequency $\omega_{b} \approx v_{\|}(r) / R_{0} q$ is assumed to be of the order of EGAM frequency, ${ }^{6}$ where $v_{\|}(r)=v_{0} \sqrt{1+\varepsilon-\lambda_{0}}$ for $\vartheta=0$. In this configuration, the quasitoroidal set of coordinates $(r, \vartheta)$ is chosen in the drift kinetic equation for the perturbed distribution function $f_{\alpha} \propto \exp (-\mathrm{i} \omega t)$ of species (electron, cold, and energetic beam ions $\alpha=e, i, b$ ),

$$
\begin{aligned}
& w \frac{\partial f_{\alpha}}{\partial \vartheta}-\mathrm{i}\left(\Omega_{\alpha}-v_{r \alpha} k_{r} \sin \vartheta\right) f_{\alpha} \\
& \quad=\frac{e_{\alpha} q R}{m_{\alpha} u} \frac{\partial F_{\alpha}}{\partial u}\left[u^{2} \frac{(2-\lambda+\varepsilon(2+\lambda) \cos \vartheta)}{2 R \omega_{c \alpha} v_{T \alpha}} E_{1} \sin \vartheta-\frac{w E_{3}}{v_{T \alpha}^{2}}\right],
\end{aligned}
$$

where the perturbations are driven by the parallel and radial components of the electric field, $E_{3}$ and $E_{1} \propto \exp (-\mathrm{i} \omega t)$. Here, $w=\sigma u \sqrt{1+\varepsilon \cos \vartheta-\lambda}, \sigma= \pm 1$, and $u=v / v_{T}$ are the parallel component and module of normalized velocities, respectively, $\lambda=v_{\perp}^{2} B_{0} /$ $B v^{2}$ is the normalized magnetic moment, $\omega_{c \alpha}=e B / c m_{\alpha}$ and $\Omega_{\alpha}$ $=\omega R_{0} q / v_{T \alpha}$ are the cyclotron and normalized wave frequencies, $v_{r \alpha}=-u^{2}(2-\lambda) q v_{T \alpha} / 2 R \omega_{c \partial}, v_{T \alpha}=\sqrt{T_{\alpha} / m_{\alpha}}$ is the thermal velocity, and $F_{e, i}$ is the Maxwell equilibrium distribution for the electrons and cold ions. The stability GAM and EGAM problems are treated within the continuum approach $k_{r}=0$, where a local continuum frequency is found from the solutions of Eq. (1) to specify the local geodesic frequency at equilibrium surfaces using electrons and cold ions in the two fluid approximations $v_{T e} \sqrt{r / R} \gg \omega R q \gg v_{T i}$. Following this model, the standard results ${ }^{16,18}$ for the parallel electric field have the following form:

$$
E_{c}=\frac{\tau_{e} \nu_{T i}}{R_{0} \omega_{c i} \Omega}\left\{2 \mathrm{i}-\mathrm{i} n_{\mathrm{h}} \frac{\nu_{T i}}{v_{0}} N_{\mathrm{s}}-\sqrt{2 \pi} \frac{\Omega^{3}}{2} \exp \left(-\frac{\Omega^{2}}{2}\right)\right\} E_{1},
$$

where $\Omega=\omega R_{0} q / v_{T i}$ and $\tau_{e}=T_{e} / T_{i}$. Here, the density perturbation response driven by the energetic ions via the coefficient $N_{\mathrm{s}} \approx O(1)$ is assumed to be small due to the small relative energetic ion density, $n_{h}=n_{0 h} / n_{0}, v_{T i} \ll v_{0}$; the NBI perturbation induced by the $E_{s}$-component is also ignored. The average radial components of the perturbed electron and ion oscillating current densities $^{16,18}$ are averaged using a general form

$$
\left\langle\tilde{j}_{r}^{\alpha}\right\rangle=e_{\alpha} v_{T \alpha}^{3} \int_{0}^{\infty} u^{2} d u \oint d \vartheta \sum_{\sigma= \pm 1} \int_{0}^{1+\varepsilon \cos \vartheta} \frac{\tilde{V}_{r, \alpha} f_{\alpha} d \lambda}{2 \sqrt{1+\varepsilon \cos \vartheta-\lambda}} .
$$

In this equation, division between the untrapped and trapped particles appears at $\lambda=1-\varepsilon$ with the NBI transit frequency, and this geodesic condition appears to be very small.

Then, taking into account the $E_{c}$-field amplitude from Eq. (2) in the sum of the components of oscillating current species ${ }^{16,18}$ driven by the geodesic wave $J_{\Sigma}=\left\langle\tilde{j}_{r}^{e}+\tilde{j}_{r}^{i}+\tilde{j}_{r}^{b}\right\rangle+j_{p}$, together with the cold ion radial oscillation current, ${ }^{19} j_{p}=-\mathrm{i} \omega c^{2} E_{1} / 4 \pi c_{A}^{2}\left[1+(3 / 4) k_{r}^{2} v_{i}^{2} / \omega_{c i}^{2}\right]$, where $c_{A}=B / \sqrt{4 \pi n_{i} m_{i}}$, the total amplitude of the oscillating radial current can be written in the following form:

$$
\begin{aligned}
J_{\Sigma}= & \frac{e_{i}^{2} n_{0} v_{0}}{m_{b} \omega_{c b}^{2} R}\left\{\frac{j_{b}}{j_{0}}+\mathrm{i} \frac{q}{\Omega} \frac{v_{T i} m_{i}}{v_{0} m_{b}}\left(\frac{7}{2}+2 \tau_{e}-\frac{\Omega^{2}}{q^{2}}\right)\right. \\
& \left.+\sqrt{\frac{\pi}{2}} \frac{v_{T i} m_{i}}{v_{0} m_{b}}\left[\frac{\Omega^{2}}{2}\left(\Omega^{2}+4 \tau_{e}+2\right) \exp \left(-\frac{\Omega^{2}}{2}\right)\right]\right\} E_{1},
\end{aligned}
$$

where $j_{0}=\frac{e_{i}^{2} n_{0} v_{0}}{m_{b} R_{0} \omega_{c b}^{2}} E_{1}$ is the normalizing coefficient and the value $j_{b}=\left\langle\tilde{j}_{r}^{b}\right\rangle$, which has to be calculated, is the averaged radial current driven in the velocity space between the injected and slowing-down beam ions. To proceed with the calculation of the oscillating energetic ion current of untrapped NBI in Eq. (3), the velocity normalization is changed to $u \equiv v / v_{0}$ and the solution of Eq. (1) is obtained using a procedure similar to that presented in Ref. 16. Generally, the $\lambda$-variable is substituted by the new variable $\kappa^{2}=2 \varepsilon /(1+\varepsilon-\lambda)$ for untrapped and $\hat{\kappa}^{2}=(1+\varepsilon-\lambda) / 2 \varepsilon$ for trapped particles in Eq. (1). The $\lambda$-integration for untrapped energetic NBI in Eq. (3) is changed to the $\kappa, x$-variables $\sin \vartheta / 2=\operatorname{sn}(\kappa, x), \cos \vartheta / 2=\mathrm{cn}(\kappa, x)$ with the Jacobi variables, ${ }^{17}$ and $\vartheta / 2=\operatorname{am}(\kappa, x)$ are introduced that transform Eq. (1) to the following form:

$$
\begin{aligned}
\frac{\partial f_{\mathrm{b}}}{\partial x} & -\sqrt{2} \mathrm{i} \sigma \frac{\Omega \kappa f_{\mathrm{b}}}{u \sqrt{\varepsilon}}-\sqrt{2} \sigma \frac{e_{b} q u F_{\mathrm{b}}^{\prime}\left(\kappa^{2}+2 \varepsilon\right)}{\sqrt{\varepsilon} \omega_{c b} m_{b} v_{0} \kappa} E_{1} \operatorname{sn}(\kappa, x) \operatorname{cn}(\kappa, x) \\
& =\frac{4 e_{b} R F_{\mathrm{b}}^{\prime}}{m_{b} v_{0}^{2}} E_{\mathrm{c}}\left(\operatorname{sn}(\kappa, x)^{2}-1 / 2\right) \operatorname{dn}(\kappa, x),
\end{aligned}
$$

where the $\vartheta$-integration is substituted by the $x$-integration between the limits $x_{ \pm}= \pm K(\kappa)$, where $K(\kappa)$ is the first kind elliptic integral, which is easily performed due to the respective sin-periodicity of the integrand. Expanding the Jacobi functions in the $Q$-series and using the periodic boundary conditions ${ }^{16-18}$ at $x= \pm K(\kappa)$, the solution of Eq. (1) driven by the $E_{1}$-component is obtained in the form

$$
f_{b}=-\mathrm{i} \frac{2 \sqrt{2} q e_{b}}{\sqrt{\varepsilon} \omega_{c b} m_{b} v_{0}} \sum_{p=1}^{N} \frac{\pi\left(\kappa^{2}+2 \varepsilon\right) Q^{p} u^{2} \Omega_{p} F^{\prime}{ }_{\mathrm{b}} E_{1}}{K(\kappa) \kappa^{3}\left(1+Q^{2 p}\right)\left(u^{2}-\Omega_{p}^{2}\right)} \sin \left(\frac{\pi p x}{K(\kappa)}\right),
$$

where $\quad \Omega_{p}=\sqrt{2} \omega R_{0} q \kappa K(\kappa) / p \pi \sqrt{\varepsilon} v_{0}, \quad Q=\left[1-\left(1-\kappa^{2}\right)^{1 / 4}\right] / 2[1$ $\left.+\left(1-\kappa^{2}\right)^{1 / 4}\right]$,

$$
\sin (\vartheta)=\sum_{p=1}^{N} \frac{4 \pi^{2} p Q}{\kappa^{2} K(\kappa)^{2}\left(1+Q^{2 p}\right)} \sin \left(\frac{\pi p x}{K(\kappa)}\right),
$$

and 


$$
\begin{aligned}
F_{\mathrm{b}}^{\prime}= & \frac{C \kappa_{0}^{3}}{16 \varepsilon u^{5}}\left\{4 \frac{u d}{\pi^{1 / 2}} \exp \left[-d^{2}\left(u-\frac{v_{s l}}{v_{0}}\right)^{2}\right]\right. \\
& -\left[6 \delta\left(\kappa-\kappa_{0}\right)+\left(\kappa^{2}+\varepsilon \kappa^{2}+2 \varepsilon\right) \frac{\kappa}{\varepsilon} \frac{\partial}{\partial \kappa} \delta\left(\kappa-\kappa_{0}\right)\right] \\
& \left.\times \operatorname{erfc}\left[\mathrm{d}\left(\frac{v_{s l}}{v_{0}}-\mathrm{u}\right)\right]\right\} .
\end{aligned}
$$

The other part of the solution driven by the $E_{c}$-component is of smaller order in comparison with the $E_{1}$-component, and it is dropped in the radial current calculation.

Further, the integration over the $\kappa$-variable can be carried out, and the results are separately presented for the normalized dissipated/unstable-emitted $P_{\mathrm{Re}}=\operatorname{Re}_{b} E_{1}^{*} /\left(j_{0} E_{1}^{*}\right)$ and the reactive $P_{\operatorname{Im}}=\operatorname{Im} J_{b} E_{1}^{*} /\left(j_{0} E_{1}^{*}\right)$ parts of the oscillating power density. Taking into account the terms of the order $O(\varepsilon)$ at the first transit resonance $\Omega_{1}<1$ and assuming $v_{\|} / v_{0}>\sqrt{2 \varepsilon}, \mathrm{d} \gg 1$, the dissipated/emitted power density is found using the slowing-down velocity $v_{s l}$ $=v_{0}\left(1-t / t_{s l}\right)$ at the beginning $t / t_{s l} \ll 1$ of the injection,

$$
\begin{aligned}
P_{\mathrm{Re}}= & -\frac{\pi^{3}(1+\varepsilon) n_{b}\left(\kappa_{0}^{2}+2 \varepsilon\right) \Omega_{1}}{256 \varepsilon^{2} \ln \left(v_{0} / v_{s l}\right) K\left(\kappa_{0}\right)^{2}} \frac{v_{\|}}{v_{0}}\left\{\left[2 \kappa_{0}^{2}-\frac{\varepsilon\left(5 \kappa_{0}^{2}+2 \varepsilon\right)}{(1+\varepsilon) \kappa_{0}^{2}}\right.\right. \\
& \left.-\frac{\left(\kappa_{0}^{4}-4 \varepsilon^{2}\right) E\left(\kappa_{0}\right)}{2 \kappa_{0}^{2}\left(1-\kappa_{0}^{2}\right) K\left(\kappa_{0}\right)}\right] \operatorname{erfc}\left[d\left(\frac{v_{s l}}{v_{0}}-\Omega_{1}\right)\right] \\
& \left.+\frac{\left(\kappa_{0}^{2}+2 \varepsilon\right)}{\sqrt{\pi}} \Omega_{1} d \exp \left[-\left(\frac{v_{s}}{v_{0}}-\Omega_{1}\right)^{2} d^{2}\right]\right\},
\end{aligned}
$$

where $\kappa_{0}=\sqrt{2 \varepsilon /\left(1+\varepsilon-\lambda_{0}\right)}=\sqrt{2 \varepsilon} v_{0} / v_{\|}$is related to the tangential surface radius of the NBI and the instability is absent $P_{\mathrm{Re}} \geq 0$ for $\Omega_{1}>1$. Here, we note that the emitted power density at the second transit resonance $\Omega_{2} \approx 2 \Omega_{1}$ is of much smaller order $P_{\mathrm{Re}}^{(2)}$ $\propto \varepsilon \Omega_{2}^{2} P_{\mathrm{Re}} / 128$ in comparison with the result in Eq. (7).

Further, using the $Z=\int_{-\infty}^{\infty} \frac{d t}{\sqrt{\pi}} \frac{\exp \left(-t^{2}\right)}{(t-x)}$-dispersion function and assuming the instability power region $v_{s l} / v_{0} \leq \Omega_{1}<1$ with $d \gg 1$, the reactive part has the following form for each surface radius that has the tangential NBI flux:

$$
\begin{aligned}
P_{\operatorname{Im}}= & \frac{\pi^{2} n_{b}\left(\kappa_{0}^{2}+2 \varepsilon\right) \Omega_{1}}{128 \varepsilon^{2} \ln \left(v_{0} / v_{s}\right) K\left(\kappa_{0}\right)^{2}} \frac{v_{\|}}{v_{0}}\left\{\left(\kappa_{0}^{2}+2 \varepsilon\right)\right. \\
& \times\left[\frac{(1+\varepsilon) \Omega_{1}}{2} d \operatorname{Re}\left\langle Z\left[\left(\Omega_{1}-v_{s} / v_{0}\right) d\right]\right\rangle\right. \\
& \left.-\frac{(1+\varepsilon) \Omega_{1}}{2\left(v_{s} / v_{0}+\Omega_{1}\right)}-\frac{\left(\Omega_{1}^{2}(1+\varepsilon)-v_{\|}^{2} / v_{0}^{2}\right)}{\left(1+2 / d-\Omega_{1}^{2}\right)}\right] \\
& +\left[2 \kappa_{0}^{2}(1+\varepsilon)-\varepsilon\left(5+\frac{v_{\|}^{2}}{v_{0}^{2}}\right)-\frac{\left(\kappa_{0}^{4}-4 \varepsilon^{2}\right) E\left(\kappa_{0}\right)}{2\left(1-\kappa_{0}^{2}\right) \kappa_{0}^{2} K\left(\kappa_{0}\right)}\right] \\
& \times\left[\ln \left(\frac{1+2 / d-\Omega_{1}^{2}}{v_{s} / v_{0}+\Omega_{1}}\right)-d\right]_{0}^{1} \ln \left|u-\Omega_{1}\right| \\
& \left.\left.\times \exp \left(-\left(u-\frac{v_{s}}{v_{0}}\right)^{2} d^{2}\right) \frac{d u}{\sqrt{\pi}}\right]\right\} .
\end{aligned}
$$

In the interval, $d\left|v_{s l} / v_{0}-\Omega_{1}\right|<1.2$, the last integral is defined by an approximate value $d \int(.) d u \mid. \approx-\ln (2 d)-\frac{\gamma_{E}}{2}+d^{2}\left(\Omega_{1}-\frac{v_{s l}}{v_{0}}\right)^{2} \operatorname{Re}$ [hipergeom $\left.\left(1,1 ; \frac{3}{2}, 2 ;-d^{2}\left(\Omega_{1}-\frac{v_{s l}}{v_{0}}\right)^{2}\right)\right]$, where $\gamma_{E} \approx 0.5772$ is the Euler constant, ${ }^{17}$ and it is well approximated by the standard asymptotic approximation $\left.d \int(.) d u\right|_{.d \rightarrow \infty} \approx \ln \left|v_{s l} / v_{0}-\Omega_{1}\right|$ for $d\left|v_{s l} / v_{0}-\Omega_{1}\right|>\sqrt{3}$.

\section{DISCUSSION}

Two sources for the EGAM instability are defined by Eq. (7) in the limit $d \gg 1$; one instability region is related to the slowing-down beam structure in the interval $v_{s l} / v_{0}<\Omega_{1}=2 \omega R_{0} q K\left(\kappa_{0}\right) / \pi v_{\|}<1$, and the other is related to a sharp peak at the distribution maximum $\propto \Omega_{1} d \exp \left[-\left(v_{s l} / v_{0}-\Omega_{1}\right)^{2} d^{2}\right]$ that is defined by the resonance condition $\Omega_{1}=v_{s l} / v_{0}$. In the first case, the instability threshold $\kappa_{0}^{2}>10 \varepsilon / 3$ appears to be similar to that, $\lambda_{0}>2 / 5$, found in Refs. 10 and 11 in the limit $\varepsilon \rightarrow 0$. Taking into account the untrapped transit effect in Eq. (7), the instability region disappears for $\varepsilon>0.17$. A simplified presentation of this instability region at $\kappa_{0}^{2} \approx 10 \varepsilon / 3$ and the phase velocity limitation $v_{\|}>2 \omega R_{0} q K\left(\kappa_{0}\right) / \pi$ are shown in Fig. 1, where the $\kappa_{0}$-dependence is changed to the normalized parallel velocity $u_{p}=v_{\|} / v_{0}=\sqrt{2 \varepsilon} / \kappa_{0}$.

In Eq. (7), the other instability at the spike resonance $\Omega_{1} \cong v_{s l} / v_{0}$ begins to have a very large value for $d \gg 1$, but this resonance moves down to the critical velocity $v_{s l} \approx V_{\mathrm{cr}}$, $V_{\mathrm{cr}} \approx 5.5 A_{h}^{1 / 3} \sqrt{T_{e} / m_{b}}$, where the $d$-value is reduced due to collisions and the dissipation part begins to be important.

In the next step, we are going to find the minimum of the continuum frequency, where the EGAM may be easily excited due to the instability defined by Eq. (7). It should be noted that the EGAM continuum frequency in Eq. (4) may be strongly modified due to the correction of the NBI transit frequency $\omega_{N B}=\pi v_{\|} /\left(2 R_{0} q K\left(\kappa_{0}\right)\right)$, where $\kappa_{0}=\sqrt{2 \varepsilon} v_{0} / v_{\|}$. In the case where the ion dissipation in Eq. (4) is very small for $\mathrm{q}>3$, the EGAM instability can be easily excited in the region shown in Fig. 1. The principal EGAM resonance is defined by the frequency condition that is slightly above the corresponding

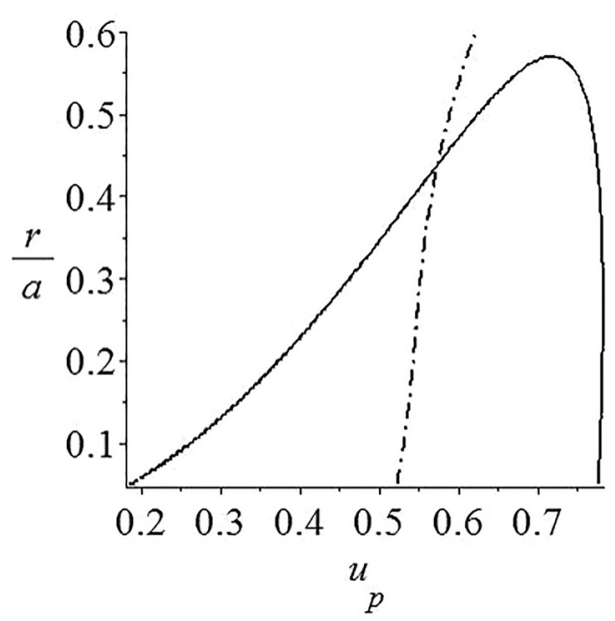

FIG. 1. Instability region defined by the radial position $r / a=3 \varepsilon$ and normalized parallel velocity $u_{p}=v_{\|} / v_{0}=\sqrt{2 \varepsilon} / \kappa_{0}$ of the energetic NBI ions. The parallel velocity limitation $v_{\| 0} / v_{\mid 0}>2 \omega R_{0} q K\left(\kappa_{0}\right) / \pi v_{\mid 0}$ is shown by dashed-dotted line for EGAM instability conditions. 
continuum minimum frequency, which may be calculated using Eqs. (4) and (8), and the frequency band has to be in the interval $v_{s l} / v_{0}<\pi \omega R_{0} q / 2 v_{\| \mid} K\left(\kappa_{0}\right)<1$. To simplify the calculations of the radial continuum distribution, the instability region is calculated at the threshold $0<\kappa_{0}^{2}-10 \varepsilon / 3 \ll \kappa_{0}^{2}$, when the $\ln (\omega)$ parts of the dispersion may be ignored in Eq. (8). In this case, the effect of the main ions in Eq. (4) becomes small and the resulting dispersion equation for the geodesic continuum becomes

$$
\begin{aligned}
& \frac{\pi n_{b}\left(v_{0}^{2}+v_{\|}^{2}\right) q^{2} \omega^{2}}{32 \ln \left(v_{0} / v_{s}\right) v_{\|}^{2} K\left(\sqrt{2 \varepsilon} v_{0} / v_{\|}\right)}\left\{\frac { ( v _ { 0 } ^ { 2 } + v _ { \| } ^ { 2 } ) } { v _ { \| } ^ { 2 } } \left[\frac{2 v_{\|}^{2} / v_{0}^{2}}{\left(1+1 / d-\Omega_{1}^{2}\right)}\right.\right. \\
& -\frac{(1+\varepsilon) \Omega_{1}}{\left(v_{s} / v_{0}+\Omega_{1}\right)}+(1+\varepsilon) \Omega_{1} d \operatorname{Re}\left\langle Z\left(\Omega_{1} d-\frac{v_{s}}{v_{0}} d\right)\right\rangle \\
& \left.-\frac{2(1+\varepsilon) \Omega_{1}^{2}}{\left(1+1 / d-\Omega_{1}^{2}\right)}\right]+\left[4(1+\varepsilon) \frac{v_{0}^{2}}{v_{\|}^{2}}-5-\frac{v_{\|}^{2}}{v_{0}^{2}}\right. \\
& \left.-\frac{\left(v_{0}^{4}-v_{\|}^{4}\right)}{v_{0}^{2}\left(v_{\|}^{2}-2 \varepsilon v_{0}^{2}\right)} \frac{E\left(\sqrt{2 \varepsilon} v_{0} / v_{\|}\right)}{K\left(\sqrt{2 \varepsilon} v_{0} / v_{\|}\right)}\right](\ln 2 d)+\frac{\gamma_{E}}{2} \\
& -d^{2}\left(\Omega_{1}-\frac{v_{s}}{v_{0}}\right)^{2} \operatorname{Re}\left[\operatorname{hipergeom}\left(1,1 ; \frac{3}{2}, 2 ;-d^{2}\left(\Omega_{1}-\frac{v_{s}}{v_{0}}\right)^{2}\right)\right] \\
& \left.+\ln \left(\frac{\left(1+1 / d-\Omega_{1}^{2}\right)}{\left(v_{s} / v_{0}+\Omega_{1}\right)}\right)\right\}+\left(\frac{7}{2}+2 \tau_{e}\right) \frac{v_{T i}^{2}}{R_{0}^{2}}-\omega^{2}=0,
\end{aligned}
$$

where $\Omega_{1}=2 \omega R_{0} q(r) K\left(\kappa_{0}(r)\right) / \pi v_{\| \mid}(r)$ depends on the radius.

Then, to demonstrate the geodesic continuum excited by the untrapped NBI ions, the radial distribution of the continuum frequency is schematically calculated using parameters that are similar to DIII-D experiments; ${ }^{6} T_{\alpha}=T_{0 \alpha}\left(1-x^{2}\right)^{2}, T_{0 e} \approx 1.0 \mathrm{keV}, \tau_{e} \approx 1.1$, with respective profiles $n_{e, i}=n_{0}\left(1-x^{2}\right), \quad x=r / a \leq 0.75$, $q=8.2\left[x^{2}(x-1.1)+0.59\right]$, with $q_{\min }=3.3$ at $x=0.7, \varepsilon=0.3 r /$ $a=0.08-0.17$, and $v_{\|} / v_{0}=115 /\left(R_{0} \pm r\right)=0.58-0.76$. As an example, Gaussian radial distributions $\propto n_{0 b} \exp \left(-2 r^{2} / a^{2}\right)$ with $n_{0 b}=(1-4) \%$ are used and the counter NBI of the untrapped and trapped particles is separated in the toroidal $(\mathrm{y}, \mathrm{R})$ and poloidal $(\mathrm{Z}, \mathrm{R})$ planes in Figs. 2(a) and 2(b).

An example of the top of the toroidal counter injection of NBI with a $210^{\circ}$ angle, trapped and untrapped trajectory between internal $\vartheta=\pi$ and external $\vartheta=0$ angles is shown in Figs. 2(a) and 2(b). The orbit width defined as the distance between the orbit point intersecting the midplane and the magnetic surface for near separator trapped and untrapped ions is defined ${ }^{6-8}$ at different theta angles $\Delta r_{t}(\vartheta=0)$ $=2 \Delta r_{u}(\vartheta=\pi)=4\left(v_{\perp}^{2} B_{0} / 2 B+v_{E}^{2} B_{0}^{2} / B_{\vartheta}^{2}\right)^{1 / 2} \varepsilon^{1 / 2} B_{0} / \omega_{c} B_{\vartheta}$.

In the respective instability region shown in Fig. 1, the EGAM continuum dependence for different NBI parallel and slowing-down velocities is presented in Figs. 3 and 4.

It should be noted that EGAM eigenmodes, which are typically formed at the continuum minimum $r / a \cong 0.1-0.2$ in Fig. 3, may have a radial structure ${ }^{4,10,19} k_{r}^{2} \rho_{L i}^{2} \approx\left(1-\omega_{E G \min }^{2} / \omega^{2}\right)$ due to a polarization current modification by the factor $\left(1-(3 / 4) k_{r}^{2} \rho_{L i}^{2}\right)$ defined by the finite Larmor radius effect or a finite orbit width effect. In the condition $\omega_{E G \min }^{2}<\omega_{N B}^{2}$ for $v_{s l} / v_{0}=0.8, v_{\|} / v_{0}=0.65$ and 0.7 , these eigenmodes may be unstable.

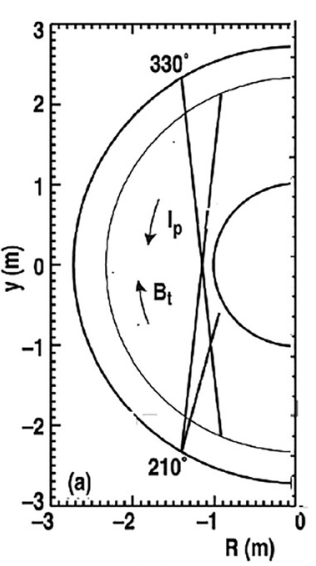

(a)

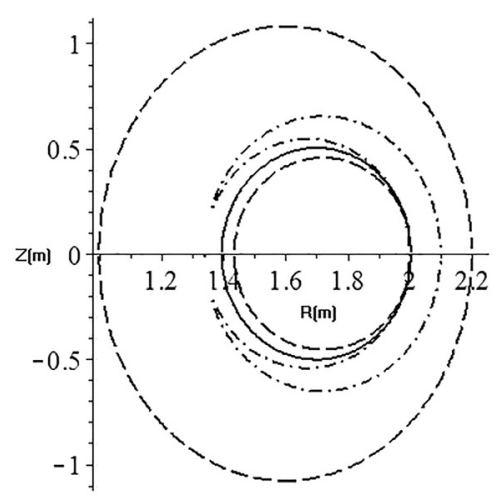

(b)
FIG. 2. (a) Schematic view from the top of the toroidal counter injection of NBI with an angle of $210^{\circ}$ and a tangential radius of $R_{\text {tng }}=1.15 \mathrm{~m}$ from the DIII-d tokamak center. (b) DIII-d poloidal cross section with the last surface (dashed lines), with solid and dashed-dotted curves showing the untrapped and trapped orbits, respectively.

Let us compare the EGAM instability at $v_{\|} / v_{0} \leq 0.75$. The increment is defined from the resonance condition $\left.\operatorname{Im} J_{\Sigma} E_{1}^{*}\right|_{\gamma=0}=0$ and the equation $\partial \operatorname{Im} J_{\Sigma} E_{1}^{*} /\left.\partial \omega\right|_{\operatorname{Im} J_{\Sigma} E^{*}=0} \gamma=\operatorname{Re} J_{\Sigma} E_{1}^{*}$ that confirms the proportionality between the decrement/increment and the density of dissipated power $\operatorname{Re} J_{\Sigma} E_{1}^{*}=j_{0} P_{\mathrm{Re}} E_{1}^{*}$. In a difference from the instability region shown in Fig. 1, the EGAM increment, which is proportional to the $P_{\mathrm{Re}}$-normalized value, appears in the region where $P_{\mathrm{Re}}<0$ that is shown in Fig. 3 for $d=10, v_{s l} / v_{0}=0.7, f=26 \mathrm{kHz}, v_{s l} / v_{0}=0.8$, and $\mathrm{f}=28 \mathrm{kHz}$. In the last case, the increment maximum at $v_{\|} / v_{0} \approx 0.74$ is related to the resonance that is slightly above the slowing-down transit frequency $\omega_{s l}=\pi v_{s l} v_{\|} / 2 v_{0} R_{0} q K\left(\kappa_{0}\right)$ and a smaller increment appears below the $\omega_{N B}$ circulation frequency.

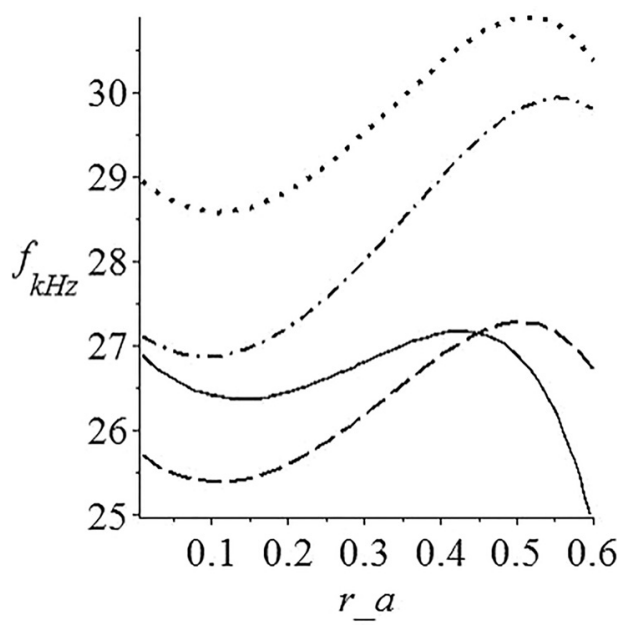

FIG. 3. Dependence of the EGAM continuum frequency over the normalized radius $r / a$ is shown for the parameters $d=10, v_{s l} / v_{0}=0.8, v_{1} / v_{0}=0.65$ and 0.7 (dotted and solid lines), $v_{s} / v_{0}=0.7, v_{\|} / v_{0}=0.7$ and 0.74 (dashed and dasheddotted lines). 


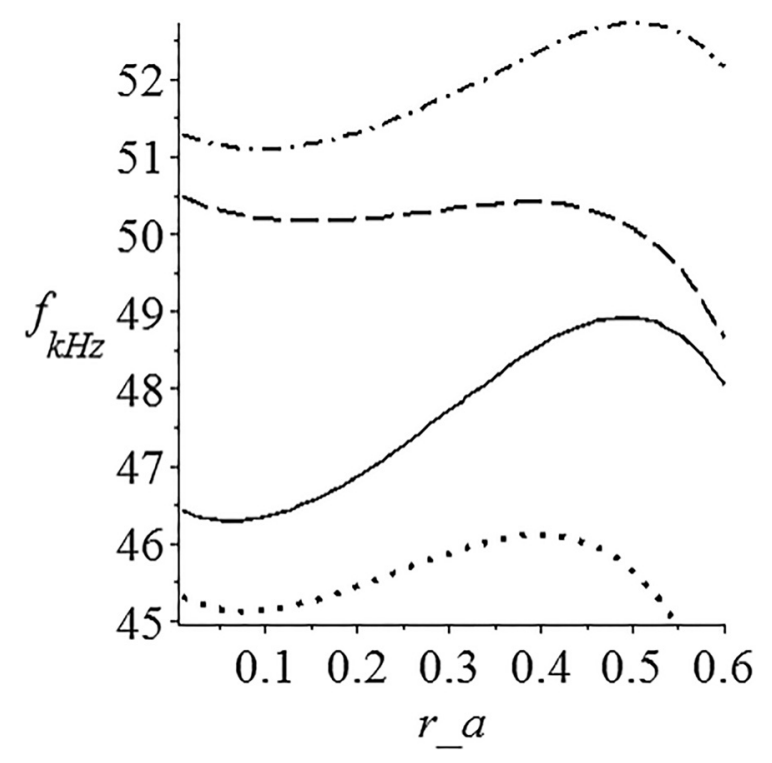

FIG. 4. Distribution of the GAM continuum frequency over the normalized radius $r / a$ is shown for the parameters $v_{s l} / v_{0}=0.8, v_{\|} / v_{0}=0.65$ and 0.7 (dotted and solid $t$ lines) and $v_{s l} / v_{0}=0.7, v_{\|} / v_{0}=0.7$ and 0.74 (dashed and dashed-dotted lines).

The standard GAM continuum remains in the stable region, and its frequency is slightly modified by the untrapped NBI, as shown in Fig. 4. In the region $r / a<0.6$, where the GAM frequency is large with respect to the NBI transit $\omega_{G A M}^{2}>\omega_{N B}^{2}$ and $P_{\mathrm{Re}} \geq 0$, this continuum frequency is calculated for the NBI density $n_{0 b}=1 \%-4 \%$ using velocity relations $v_{s l} / v_{0}=0.8, v_{\|} / v_{0}=0.65,0.7$ and $v_{s l} / v_{0}=0.7$, $v_{\|} / v_{0}=0.74$.

\section{SUMMARY}

As mentioned in the Introduction, we first note new features in our model that consider the situation in which the geodesic phase resonance may appear well above the critical velocity, where the energetic ions begin to be substantially diffused/scattered, which form the bump distribution. The minority NBI distribution is modeled by the energetic ion tail with the maximum in the untrapped-passing region that remains between a magnetic axis and the trapped NBI boundary surface. Using the method of Jacobi functions, the drift kinetic equation for the perturbed distribution function of the untrapped energetic ions is analytically solved and the geodesic mode spectrum is found from the zero condition for the perturbed total radial current, where bulk plasma particles are treated in the fluid limit. The difference in the physics of the geodesic modes is determined by the boundary that is formed by the untrapped NBI transit frequency $\omega_{N B}=\pi v_{\|} /$ $\left(2 R_{0} q K\left(\sqrt{2 \varepsilon} v_{0} / v_{\|}\right)\right)$depending on the pitch angle, and the stable GAM remains above and the unstable EGAM can appear below the $\omega_{N B}$-frequency. The maximum value of EGAM increments is found to be in the frequency range that is slightly above the slowing-down transit frequency $\omega_{s}=\omega_{N B} v_{s} / v_{0}$. Physically, the result of these calculations is similar to that observed in the DIII-d experiments ${ }^{6}$ and substantially different from EGAM instability conditions driven by trapped hot ions during ICR heating experiments in JET tokamaks. ${ }^{4,16,18}$ It should be noted that the standard GAMs are stable and their frequency is only slightly modified by the energetic NBI ion tail.

\section{ACKNOWLEDGMENTS}

This work was supported by Conselho Nacional de Desenvolvimento Cientifico e Tecnológico (CNPq), Brazil, Award/ Contract Number 307984/2016-8, and by Princeton Plasma Physics Laboratory, Award/Contract Number DE-AC02-09CH11466.

\section{REFERENCES}

${ }^{7}$ V. B. Lebedev, P. N. Yushmanov, P. H. Diamond, S. V. Novakovskii, and A. I. Smolyakov, Phys. Plasmas 3, 3023 (1996).

${ }^{2}$ A. V. Melnikov, L. G. Eliseev, S. V. Perfilov, S. E. Lysenko, R. V. Shurygin, V. N. Zenin, S. A. Grashin, L. I. Krupnik, A. S. Kozachek, R. Yu. Solomatin, A. G. Elfimov, A. I. Smolyakov, and M. V. Ufimtsev, Nucl. Fusion 55, 063001 (2015).

${ }^{3}$ G. D. Conway, C. Angioni, F. Ryter, P. Sauter, and J. Vicente, Phys. Rev. Lett. 106, 0655001 (2011).

${ }^{4}$ H. L. Berk, C. J. Boswell, D. Borba, A. C. A. Figueiredo, T. Johnson, M. F. F. Nave, S. D. Pinches, and S. E. Sharapov, Nucl. Fusion 46, S888 (2006).

${ }^{5}$ G. R. McKee, D. K. Gupta, R. J. Fonck, D. J. Schlossberg, M. W. Shafer, and P. Gohil, Plasma Phys. Controlled Fusion 48, S123 (2006).

${ }^{6}$ R. Nazikian, G. Y. Fu, M. E. Austin, H. L. Berk, R. V. Budny, N. N. Gorelenkov, W. W. Heidbrink, C. T. Holcomb, G. J. Kramer, G. R. McKee, M. A. Makowski, W. M. Solomon, M. Shafer, E. J. Strait, and M. A. Van Zeeland, Phys. Rev. Lett. 101, 185001 (2008).

${ }^{7}$ R. K. Fisher, D. C. Pace, G. J. Kramer, M. A. Van Zeeland, R. Nazikian, W. W. Heidbrink, and M. Garcia-Munoz, Nucl. Fusion 52, 123015 (2012).

${ }^{8}$ A. A. Galeev and K. Z. Sagdeev, Sov. Phys. JETP 26, 223 (1968) [Zh. Exp. Teor. Fiz. 53, 348 (1967)].

${ }^{9}$ V. Lakhin and E. Sorokina, Phys. Lett. A 378, 535 (2014).

${ }^{10}$ G. Y. Fu, Phys. Rev. Lett. 101, 185002 (2008).

${ }^{11}$ Z. Qiu, F. Zonca, and L. Chen, Plasma Phys. Controlled Fusion 52, 095003 (2010).

${ }^{12}$ T. H. Stix, Plasma Phys. 14, 367 (1972).

${ }^{13}$ N. N. Gorelenkov, H. L. Berk, and R. V. Bundy, Nucl. Fusion 45, 226 (2005).

${ }^{14}$ Z. S. Qu, M. J. Hole, and M. Fitzgerald, Phys. Rev. Lett. 116, 095004 (2016).

${ }^{15}$ D. Zarzoso, D. del-Castillo-Negrete, D. F. Escande, Y. Sarazin, X. Garbet, V. Grandgirard, C. Passeron, G. Latu, and S. Benkadda, Nucl. Fusion 58, 106030 (2018).

${ }^{16}$ A. G. Elfimov, Phys. Plasmas 25, 062512 (2018).

${ }^{17}$ M. Abramowitz and I. A. Stegun, Handbook of Mathematical Functions (Dover, 1972).

${ }^{18}$ A. G. Elfimov, Phys. Lett. A 378, 3533 (2014).

${ }^{19}$ S. I. Itoh, K. Itoh, M. Sasaki, A. Fujisawa, T. Ido, and Y. Nagashima, Plasma Phys. Controlled Fusion 49, L7-10 (2007). 